\title{
Necrosis of the Bone Marrow in Acute Pancreatitis
}

In the course of acute pancreatitis fat necroses develop not only in the pancreas and peripancreatic fat but frequently also in mesenterial and peritoneal fat. The formation of extraabdominal fat necroses is less frequent. Recently it was found that in the course of acute pancreatitis sometimes also fat necroses of the bone marrow develop (Immelman et al.: Amer. J. Med. 36: 96 [1964]; Gerke et al.: Radiology 85: 330 [1965]).

The pathogenesis of fat necroses of the bone marrow has not been reliably established. The action of activated lipase is assumed. Fat necroses of the bone marrow were observed in man as well as in experimental animals (Scarpellí et al.: Amer. J. Path. 32: 1077 [1965]), i. e. mainly when in the course of pancreatitis numerous intra- and extraabdominal necroses developed. So far no relationship was revealed between the formation of fat necroses of the bone marrow and the concentration of pancreatic enzymes in the blood, the duration or severity of clinical manifestations of acute pancreatitis.

Fat necrosis of the bone marrow which developed in the course of acute pancreatitis either does not manifest itself at all clinically or causes pain in the affected portions of bones, usually pain of a mild intensity. The site of osseous changes are either the ends of long bones, above all the distal end of the femur or proximal end of the tibia -so-called metadiaphyseal type of necrosis or the epiphyseal parts of long bones, mainly the head of the femur and humerus - so-called epiphyseal type of necrosis.

Fat necrosis of the bone marrow may cause infarction of the bone marrow. Three mechanism may be involved in the participation of the inflammatory affection of the exocrine pancreas in the development of ischaemia of the bone marrow and thus also infarction.

(1) The long wound intramedullary arteries taking sharp bends may become, due to changes in the bone marrow caused by the fat necrosis, compressed or distorted. Thus aseptic necrosis or infarction develop. Bucly (Brit. J. Radiol. 32: 22 [1959]) found that infarction of the bone marrow develops more frequently in bones with this vascular pattern.

376

Editorial

The development of vascular necrosis in the vicinity of the fat necrosis of the marrow may cause ischæmia and the development of infarction.

Ischæmia of the bone marrow may be caused also by fat embolism into the íntermedullary arteríoles, in particular in patients with hyperlipæmia - permanent or temporary - which developed in the course of acute pancreatitis. All six patients with intermedullary fat necrosis observed in the course of acute pancreatitis by Gerke et al. and three of a total of four patients observed by Immelman et al. were alcoholics. Repeatedly attention has been drawn to the relationship between excessive alcohol consumption and the development of hyperlipæmia. ScarpelWs finding of $10.4 \%$ macroscopically detectable fat necroses of the bone marrow in 63 cases of acute necrosis of the pancreas sub jected to necropsy, suggested that metastatic fat necroses of the bone marrow are more frequent in the course of acute pancreatitis than 
generally assumed. This is probably due to the fact that their occur rence in the course of acute pancreatitis is so far not generally known and therefore they escape attention. K. Herfort, Prague 